\title{
Effect of Oil Pulling on Plaque and Gingivitis
}

\author{
HV Amith, Anil V Ankola, L Nagesh
}

\section{ABSTRACT}

Oil pulling is an age-old process mentioned in Charaka Samhita and Sushratha's Arthashastra. This study was conducted to assess the effect of oil pulling on Plaque and Gingivitis.

Objectives: (1) To assess the effect of oil pulling on plaque and gingivitis. (2) To monitor its safety on oral soft and hard tissues.

Methodology: 10 subjects performed Oil Pulling along with their other oral hygiene measures for 45 days, using Refined Sunflower Oil. Their Plaque and gingival scores were assessed periodocally by modified PHP and Gingival indices. The results were subjected to t-test and Wilcoxon signed rank test.

Results: The reduction in plaque and gingival scores from baseline to 45 days were $0.81+-0.41(p<0.01)$ and $0.39+0.17(p<0.01)$ respectively. The differences were found to be statistically significant.

Conclusion: Oil pulling is having dental benefits. Hence this holds a chance to be added to other oral hygiene measures.

\section{Contact Author}

\section{Dr. HV Amith}

E-mail : amith_hv@yahoo.co.in

Key words: Plaque; Gingivitis; Rinsing; Oil pulling.

$\mathrm{T}$ The interrelationship between oral and general health is proven by evidence. Severe periodontal disease, for example, is associated with diabetes. The strong correlation between several oral diseases and noncommunicable chronic diseases is primarily a result of the common risk factors.(1) Many general disease conditions also have oral manifestations that increase the risk of oral disease which, in turn, is a risk factor for a number of general health conditions. This wider meaning of oral health does not diminish the relevance of the two globally leading oral afflictions - dental caries and periodontal diseases. Both can be effectively prevented and controlled by an effective plaque control method. Daily plaque removal with a toothbrush is an important component of most oral hygiene programs intended to prevent and treat periodontal diseases. It has been clearly demonstrated that when properly used, both manual and electric toothbrushes are effective in removing supragingival plaque and reducing clinical signs of gingival inflammation.(2) Mechanical tooth cleaning even today remains the most reliable method of controlling supragingival bacterial plaque. Failure to comply and lack of technical skill of the patient has lessened the effectiveness of conventional tooth brushing.(3) Because traditional mechanical methods for controlling plaque have proven inadequate, research efforts have focused on chemotherapeutic agents for reducing or preventing plaque induced oral diseases.(4) No single agent can perfectly get rid of dental plaque. Depending on the situation, various combinations of agents have been tried.

Oil pulling is an age-old process mentioned in Charaka Samhita and Sushrutha's Arthashastra. It's a widely recommended procedure in Ayurveda. The process is called Kavala Gandoosha/ kavala Graha in Ayurveda.(5) In Ayurveda this process is said to cure about 30 systemic diseases ranging from headache, migraine to hypertension, diabetes, asthma etc. It basically slows down the ageing process.(5)

There has been no mention of studies on "Oil pulling" in the dental literature. A specific type of oil pulling called "Roopana Gandoosha" has been mentioned in Ashtanga Sangraha and is said to have dental benefits.(6,7) Various oils like Refined Sunflower oil, Sesame oil, Olive oil etc can be used for Oil Pulling. Liquids from milk and water to extracts of Ghooseberries and mangoes have been used for oil pulling.(8)

In view of the unavailability of a household agent which can comprehensively prevent plaque induced oral diseases, this study was initiated to assess the dental benefits of Oil pulling. 
The present study aimed to assess the effect of Oil pulling on oral hygiene. The objectives of the study were to assess the effect of oil pulling on plaque and gingivitis, to monitor its safety on oral soft and hard tissues and to assess the acceptability of Oil pulling.

\section{Methodology}

After screening the entire batch of 50 first year Physiotherapy students, at the KLES's Physiotherapy College, Belgaum, 10 male students in the age range 19-21 years were recruited for a 45 days study. The criteria for selection of the subjects were:

\section{Inclusion criteria}

- Subjects willing to participate.

- Subjects having at least 20 natural teeth in the permanent dentition.

- Subjects with mild to moderate gingivitis and plaque accumulation.

- Subjects willing to refrain from any form of dental treatments during the study period.

\section{Exclusion criteria}

- Subjects having allergy to the oil used.

- Subjects with systemic diseases and using antibiotics.

- Subjects undergoing orthodontic treatment or using intraoral artificial prosthesis.

- Subjects using any other mouth wash / rinse.

\section{Armamentarium}

- Refined sunflower oil (Fortune Company)

- Measurement beaker

- Containers and tea spoons

- Mouth mirror

- Probe

- Tweezers

- Cotton

- Kidney tray

- Two tone Disclosing agent (Alpha plac).

\section{Pre-study procedures}

- The subjects were blinded about the aim of the investigation in order to avoid any possible bias.

- In order to participate the subjects signed a witnessed consent form and committed themselves to the study.

- Ethical clearance was obtained from the KLE Society Ethical committee.

\section{Experimental Protocol}

This study was carried out at the Department of Preventive and Community Dentistry.

Oral prophylaxis was not performed so that the subjects began the treatment regimen with their normal existing level of plaque deposits. All subjects were instructed to continue their normal home oral hygiene procedures, along with Oil Pulling. All subjects had to perform their routine morning Oil Pulling prior to each of the clinical examination on the experimental days. Plaque levels and the severity of Gingivitis were assessed on day 0 (baseline- $\mathrm{T}_{0}$ ). The subjects were called for 3 subsequent visits, spaced 15 days apart. At each visit, plaque and gingivitis scores were assessed.

- $\mathrm{T}_{1}$ - After 15 days.

- $\mathrm{T}_{2}$ - After 30 days.

- $\mathrm{T}_{3}$ - After 45 days.

All the subjects were required to follow the below mentioned, recommended method of Oil Pulling.

\section{The oil pulling procedure}

Take 10-15 ml of refined sunflower oil using a tea spoon, approximately 6 gms or till the mouth is half filled. Sip, suck and pull the oil through the teeth. Lift your chin a bit, close eyes and start swishing liquid from left to right, front to back and vice versa. Concentrate and imagine liquid moving inside the mouth. Swish approximately 8-10 minutes or till you feel a fullness in your mouth. At the end the oil should be milky white, thin and frothy. Spit the liquid.

\section{Plaque Scoring}

Patient Hygiene Performance Index developed by Podshadley AG and Haley JV (1968) was modified to score the plaque from both the buccal and lingual / palatal surface of all the teeth instead of just the Index teeth. For assessment, the plaque was disclosed using Two tone disclosing agent.(9) The disclosing agent was applied with cotton and rinsed after 1 minute. Each tooth surface (facial and lingual) was divided into 5 sections by imaginary lines (Figure 1):

- Mesial third

- Distal third

- Middle third further divided horizontally into gingival, middle and occlusal sections as shown below.

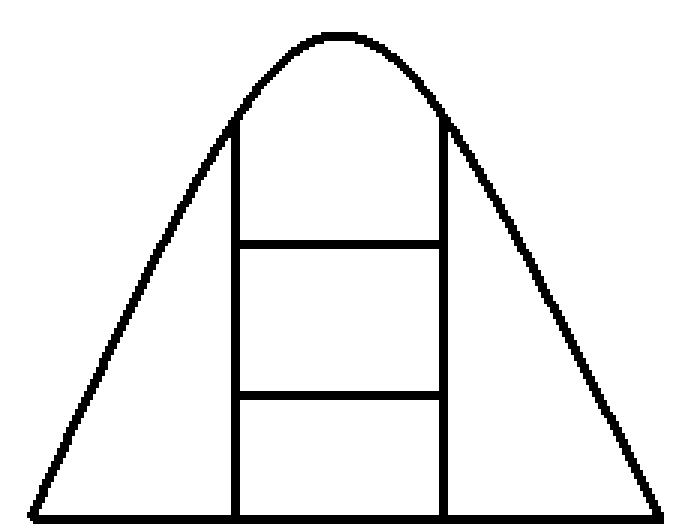

Fig. 1: Division of tooth surface to record PHP index 


\section{Scores}

- 0 - Absence of Plaque

- 1 - Presence of Plaque

The score was calculated by adding all the values for each sub-division on both buccal and lingual for each tooth. The total score was divided by the number of teeth examined to get a individual score. It was interpreted as follows:
- Excellent $=0$
- Good $=0.1-1.7$
- Fair $=1.8-3.4$
- Poor $=3.5-5.0$

\section{Gingivitis Scoring}

Gingival index by Loe H and Sillness J (1963) was used to assess the severity of Gingivitis on the index teeth 16, 12, 24, $36,32,44$. The tissues surrounding each tooth were divided into four gingival scoring units, distal-facial papilla, facial margin, mesial-facial papilla and the entire lingual gingival margin. Ablunt instrument, such as a periodontal pocket probe, was used to assess the bleeding potential of the tissues.(10)

\section{Scores}

- 0 - Absence of inflammation / normal gingival

- 1 - Mild inflammation, slight change in color, slight edema, no bleeding on probing.

- 2 - Moderate inflammation, moderate glazing, redness, edema and hypertrophy, bleeding on probing.

- 3-Severe inflammation, marked redness and hypertrophy, ulceration, tendency to bleed spontaneously.

Totaling the scores around each tooth gave the gingival index score for the area. Totaling all the scores and dividing by the number of teeth examined provided the gingival index score for that person, which was interpreted as follows:

- Mild gingivitis $=0.1-1.0$
Moderate gingivitis $=1.1-2.0$

Severe gingivitis $=2.1-3$

A single examiner performed all the clinical measurements at the same time of the day throughout the study. Reproducibility was assessed by rescoring 5 teeth randomly at each examination. The intra-examiner reproducibility for plaque scoring was 0.81 and for gingival index was 0.75 .

\section{Statistical Analysis}

Students paired ' $t$ ' test and Wilcoxon signed rank test were used to compare the plaque and gingivitis scores before and after performing Oil pulling. The validity level was fixed to $\mathrm{p}<0.05$.

\section{Acceptability of Oil pulling}

This was assessed at the end of the study period of 45 days. Each subject was given a close ended self administered questionnaire consisting of 15 questions to check for the acceptability of Oil pulling.

\section{Results}

The present study was conducted to assess the dental benefits of Oil pulling. Plaque and Gingival indices were performed on the subjects at baseline, 15, 30 and 45 days of Oil pulling. The changes in the index scores during the study period were compared.

No adverse reactions to any hard and soft tissues of the oral cavity were noticed during the study. The resultant changes in the plaque scores have been summarized in Tables 2, accompanied by Figure 2. There was a net decline in mean plaque scores from baseline to 45 days amounting to $0.81 \pm 0.41$ $(\mathrm{p}<0.01)$. The greatest reduction in plaque scores was noted at the examinations between 15 and 30 days (0.37). Though the decline in plaque scores at 15 days was not significant, the rest of the differences were highly significant.

\section{Table 1: Effect of oil pulling on plaque and gingiva}

\begin{tabular}{|c|c|c|c|c|}
\hline Serial no. & Baseline & 15 days & 30 days & 45 days \\
\hline 1. & $3.27^{*} 0.21^{\#}$ & $3.21 \underline{0.21}$ & 3.140 .17 & 3.050 .08 \\
\hline 2. & 3.640 .79 & 3.590 .75 & 3.430 .58 & 2.960 .46 \\
\hline 3. & 3.470 .38 & 3.200 .25 & $\underline{3.230} 0.21$ & 3.090 .17 \\
\hline 4. & 3.450 .79 & $\underline{3.580 .50}$ & 3.190 .42 & 2.960 .25 \\
\hline 5. & 3.440 .46 & 3.060 .33 & 2.850 .25 & 2.270 .17 \\
\hline 6. & 3.140 .79 & 2.980 .71 & $\underline{3.120} 0.63$ & 2.730 .42 \\
\hline 7. & 3.371 .04 & $\underline{3.650} 0.88$ & 2.520 .75 & 2.180 .50 \\
\hline 8. & 3.340 .63 & 3.210 .54 & 2.530 .46 & 2.230 .29 \\
\hline 9. & 3.560 .88 & $\underline{3.750} 0.75$ & 2.940 .58 & 2.440 .38 \\
\hline 10. & 3.901 .21 & 3.611 .0 & 3.230 .75 & 2.610 .54 \\
\hline Mean \pm Std Dev. & $3.46 \pm 0.210 .72 \pm 0.30$ & $3.38 \pm 0.260 .59 \pm 0.30$ & $3.02 \pm 0.310 .48 \pm 0.21$ & $2.65 \pm 0.360 .33 \pm 0.16$ \\
\hline
\end{tabular}


Table 2: Comparison of plaque scores between baseline, 15, 30 and 45 days

\begin{tabular}{|c|c|c|c|c|c|c|c|}
\hline Period & Mean & Std.Dev. & MeanDiff. & Std.Dv.Diff. & Paired t-value & P-value & Significance \\
\hline Base line & 3.4580 & 0.2102 & & & & & \\
\hline 15 days & 3.3840 & 0.2787 & 0.0740 & 0.2177 & 1.0748 & 0.3104 & NS \\
\hline Base line & 3.4580 & 0.2102 & & & & & \\
\hline 30 days & 3.0180 & 0.3046 & 0.4400 & 0.3001 & 4.6363 & 0.0012 & $S$ \\
\hline Base line & 3.4580 & 0.2102 & & & & & \\
\hline 45 days & 2.6520 & 0.3558 & 0.8060 & 0.4086 & 6.2378 & 0.0002 & $S$ \\
\hline 15 days & 3.3840 & 0.2787 & & & & & \\
\hline 30 days & 3.0180 & 0.3046 & 0.3660 & 0.4010 & 2.8860 & 0.0180 & $S$ \\
\hline 15 days & 3.3840 & 0.2787 & & & & & \\
\hline 45 days & 2.6520 & 0.3558 & 0.7320 & 0.4696 & 4.9292 & 0.0008 & $S$ \\
\hline 30 days & 3.0180 & 0.3046 & & & & & \\
\hline 45 days & 2.6520 & 0.3558 & 0.3660 & 0.1796 & 6.4428 & 0.0001 & S \\
\hline
\end{tabular}

The changes in the gingivitis scores have been summarized in Tables 3, accompanied by Figure 3. The net decline in gingivitis was $0.39 \pm 0.17(\mathrm{p}<0.01)$. The decrease in gingivitis was more than $50 \%$.

The study consisted of 10 male subjects, who were assessed for plaque and gingivitis at baseline, 15, 30 and 45 days. On

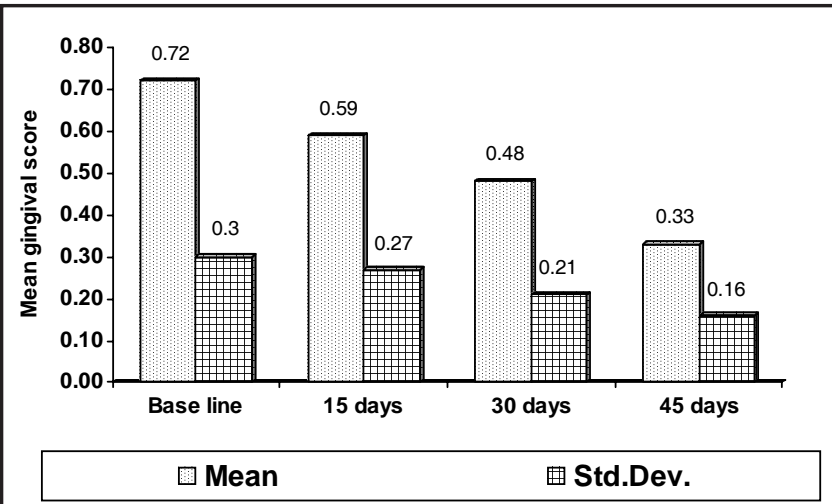

Fig. 2: few occasions there have been increase in individual plaque scores as shown by the underlined scores, but there was a net decline in the mean plaque scores throughout 45 days. On one occasion the gingivitis score for one subject remained constant from baseline to 15 days. But even the gingival index scores showed a declining trend from baseline to 45 days. The mean plaque scores from baseline to 15,30 and 45 days were $3.46 \pm$ $0.21,3.38 \pm 0.26,3.02 \pm 0.31$ and $2.65 \pm 0.36$ respectively. The mean gingivitis scores from baseline to 15,30 and 45 days were $0.72 \pm 0.30,0.59 \pm 0.30,0.48 \pm 0.21$ and $0.33 \pm 0.16$ respectively.

At baseline $\left(\mathrm{T}_{0}\right)$ the mean plaque score and Standard Deviation was 3.46 \pm 0.21 . After 15 days $\left(\mathrm{T}_{1}\right)$ the mean plaque score and $\mathrm{SD}$ was 3.38 \pm 0.28 . After 30 days $\left(\mathrm{T}_{2}\right)$ the mean plaque score and $\mathrm{SD}$ was 3.02 \pm 0.30 . After 45 days $\left(\mathrm{T}_{3}\right)$ the mean plaque score and SD was $2.65 \pm 0.36$. There was a net decline in plaque scores from baseline to 45 days. Except for the decrease in plaque scores from $\mathrm{T}_{0}$ to $\mathrm{T}_{1}$, rest all comparisons were highly significant. The highest reduction being between baseline to 45 days, (3.46 to 2.65) with a p value of 0.0002 .

Table 3: Comparison of gingivitis scores between baseline, 15, 30 and 45 days

\begin{tabular}{|c|c|c|c|c|c|c|c|}
\hline Period & Mean & Std.Dv. & MeanDiff. & Std.Dv.Diff. & Paired t-value & P-value & Signi. \\
\hline Base line & 0.7180 & 0.3044 & & & & & \\
\hline 15 days & 0.5920 & 0.2700 & 0.1260 & 0.0829 & 4.8068 & 0.0010 & S \\
\hline Base line & 0.7180 & 0.3044 & & & & & \\
\hline 30 days & 0.4800 & 0.2145 & 0.2380 & 0.1199 & 6.2777 & 0.0001 & $S$ \\
\hline Base line & 0.7180 & 0.3044 & & & & & \\
\hline 45 days & 0.3260 & 0.1571 & 0.3920 & 0.1673 & 7.4087 & 0.0000 & $S$ \\
\hline 15 days & 0.5920 & 0.2700 & & & & & \\
\hline 30 days & 0.4800 & 0.2145 & 0.1120 & 0.0671 & 5.2758 & 0.0005 & $S$ \\
\hline 15 days & 0.5920 & 0.2700 & & & & & \\
\hline 45 days & 0.3260 & 0.1571 & 0.2660 & 0.1190 & 7.0689 & 0.0001 & S \\
\hline 30 days & 0.4800 & 0.2145 & & & & & \\
\hline 45 days & 0.3260 & 0.1571 & 0.1540 & 0.0682 & 7.1424 & 0.0001 & $S$ \\
\hline
\end{tabular}




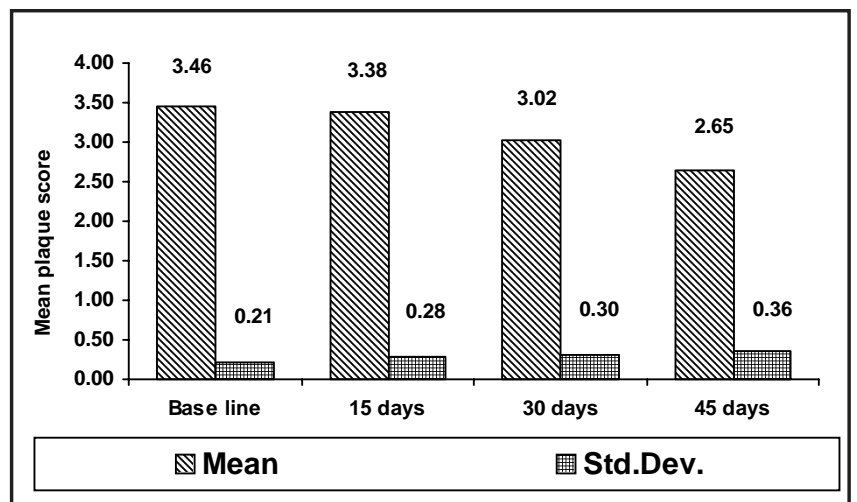

Fig. 2:

At baseline $\left(\mathrm{T}_{0}\right)$ the mean gingivitis score and $\mathrm{SD}$ was $0.72 \pm 0.30$. After 15 days $\left(\mathrm{T}_{1}\right)$ the mean gingivitis score and SD was $0.59 \pm 0.27$. After 30 days $\left(\mathrm{T}_{2}\right)$ the mean gingivitis score and SD was $0.48 \pm 0.21$. After 45 days $\left(\mathrm{T}_{3}\right)$ the mean gingivitis score and $\mathrm{SD}$ was $0.33 \pm 0.16$. There was a net decline in gingivitis scores from baseline to 45 days. All the comparisons from $\mathrm{T}_{0}$ to $\mathrm{T}_{3}$ were highly significant. The highest reduction being between $T_{0}$ to $T_{3}(0.7180$ to 0.3260$)$. Unlike the plaque scores, not even in a single instance, was there a rise in the gingival index scores throughout the study period.

The plaque and gingivitis scores between baseline, 15 days, 30 days and 45 days were compared by a non parametric testthe Wilcoxon Matched Pairs Test also. Similar results have been obtained even in this instance. Except for the decrease in plaque scores from $\mathrm{T}_{0}$ to $\mathrm{T}_{1}$, rest all comparisons were highly significant. All the comparisons for gingivitis reduction from $\mathrm{T}_{0}$ to $\mathrm{T}_{3}$ were highly significant.

\section{Acceptability of Oil pulling}

The acceptability of Oil pulling was assessed by a self administered questionnaire, the results of which are as follows:

\section{Discussion}

An exhaustive search of literature didn't yield any studies related to Oil Pulling and oral health. This is an indigenous procedure, having its origin in the Aurvedic literature of India. Oil Pulling has been mentioned in Charakasamhita and Sushrata's Arthashastra. For this reason, by no means Oil Pulling deserves a lack of scientific interest. In spite of most of the Ayurvedic texts stressing the systemic benefits of Oil Pulling, our study has proved, it's indeed having dental benefits also.

Since there are no studies till date assessing the dental benefits of Oil Pulling, our study has been the first in this untrodden path. When compared to tooth brushes and mouth rinses, Oil Pulling has also resulted in a significant improvement in oral hygiene.

Though refined sunflower oil has been used in the present study, there has been mention of other oils like sesame oil, groundnut oil, olive oil, mustard oil etc. Liquids from water and milk to extracts of gooseberries have been used for Oil Pulling. Some oils are more palatable than others in their raw state. Hence the author after trying various types of oils, came to a conclusion to use the most palatable, the refined sunflower oil for this study.

Though Oil Pulling has resulted in a significant reduction in Plaque and gingivitis, it can't be considered as a replacement for tooth brushing, but can definitely be a supplemental oral hygiene aid. The most objectionable part of this procedure is that, it has to be performed early in the morning, on empty stomach. Though all the subjects in this study complied with this during the study period, it can't be assured that they will perform this procedure lifelong. The results of the questionnaire indicated that $80 \%$ of the study subjects were willing to perform this procedure lifelong. It takes a lot of determination on part the subjects to master this procedure and perform it on a regular basis. Unless and until the subjects are sufficiently motivated, Oil Pulling can be rarely voluntarily accepted. More over if the subjects are disabled, handicapped, its difficult for them to adopt this procedure.

The most wonderful part of Oil Pulling is that, it can be performed using any oil easily available at homes. Refined sunflower oil or any other cooking oil for that matter is a common house hold commodity in most of the Indian homes. Hence the material for oil pulling is easily accessible to most of the Indian population right at their homes. This is a therapy that can be practiced right at home, without any expenses, and has a huge storehouse of benefits.(11)

A liter of any brand of refined oil at present costs about 55-70 rs on an average. Whereas most of the available mouth rinses cost about 30-40 rs per $100 \mathrm{ml}$. If the cost per rinse is compared, Oil Pulling will cost about 0.55 to 0.70 rs per rinse, whereas a mouthrinse will cost 2.50 to 3.00 rs.

The Oil Pulling procedure has to be performed in a calm and bright area, early in the morning. How many of us can afford to spare 10 to 15 minutes of our early morning time, to perform Oil Pulling, by sitting calmly and concentrating the oil inside our mouth. In the context of the present day busy lives, its very hard to accept this procedure, unless and until more studies are carried out, and the benefits are proven repeatedly.

According to Ayurvedic literature Oil Pulling has been known to be effective on some 30 systemic diseases. Such a precious and practical solution to so many problems, has remained elusive to our sight since a long time. So this study is an eye opener to encourage further studies in this regard.

Chlorhexidine-containing mouth washes have been widely used 
as clinical adjunct in the treatment of both caries and periodontal diseases providing a 'gold standard' by which to assess the efficacy of other topically applied agents. Chlorhexidine may discourage compliance because of its unpleasant taste and undesirable side effects such as tooth staining and alterations in taste sensations. Similarly stannous containing products have been associated with extrinsic stain formation, and both stannous and zinc salts have organoleptic problems that restrict the concentrations that can be used. No such adverse reactions were noted during this study.

No reports have been mentioned in the dental literature about studies on oil pulling though this was a widely practiced procedure. Khalessi AM et al. conducted a study to assess the oral health efficacy of Persica mouthwash containing an extract of S. persica, and found that it resulted in a $20-26 \%$ reduction in plaque accumulation.(12) This is in accordance with our study, where we also have noted reduction in plaque index scores after oil pulling.

In a study by Putt MS et al assessing the efficacy of alum containing mouth rinse, $22 \%$ reduction in plaque and $13 \%$ reduction in gingivitis was noted. At both 2 and 4 week examinations, the alum group had lower plaque thickness scores than the placebo group, but neither reduction attained statistical significance. In the present study the reduction of plaque scored from baseline to 15 days was not significant, whereas rest all reductions were statistically significant.

According to Tooth brush studies by Stoltze K the reduction in percentage sites with visible plaque was $8 \%$ in the manual toothbrush group and 30\% in the Electric toothbrush group.(13) For approximal sites the corresponding figures were $10 \%$ and $35 \%$. The mean reduction in gingivitis were $8 \%$ and $9 \%$ after 6 weeks, with all sites, whereas for the approximal sites reductions were $9 \%$ and $11 \%$ respectively. The results are in concurrence with our study, except for the fact that Oil Pulling has resulted in a far greater reduction in gingivitis, than the manual or electric tooth brushes.

In a study by Tritton $\mathrm{CB}$ and Armitage $\mathrm{GC}(3)$ tooth brushing has reduced plaque scores by $11-27 \%$ and gingivitis by $8-23 \%$. By oil pulling, in the present study plaque scores have reduced by $18-30 \%$ and gingivitis has reduced by $52-60 \%$. Hence reduction in plaque is comparable to previous studies, whereas reduction in gingivitis has been far superior. Being an indigenous procedure of Ayurveda this has a wide scope if properly utilized.

One important factor which requires discussion at this juncture is the "Hawthorne effect"- a positive change in the behaviour of a subject as a result of the special attention and status received from participation in an investigation.(14) Similarly it has been observed that oral hygiene may improve during a clinical trial as a result of anticipation of oral examinations which occur as part of study participation.(15) We agree that this effect might have played its part in our present study, which was of a short duration. If not elimination, the only way by which we could have minimized Hawthorne effect was by increasing the duration of the study.

In the light of the present study it is concluded that Oil Pulling is having dental benefits and it has resulted in a significant reduction in plaque and gingivitis scores. Hence it can be recommended as an Oral hygiene aid.

\section{Conclusion}

Within the limits of the present study, it can be concluded that Oil Pulling has the ability to reduce plaque and gingivitis. It emerges from this study that an individual is able to maintain low levels of plaque and gingivitis even if he is performing Oil Pulling, just for 45 days.

This study is just the tip of an iceberg and we recommend that further longitudinal studies with larger sample size should be conducted. Research comparing the use of different types of oils for the Oil Pulling procedure, and their effect on oral hygiene are needed. Also microbiological analysis of saliva and plaque if considered will add more internal validity to such studies. Further, studies comparing the effect of various commonly prescribed mouthwashes and Oil Pulling on the oral hygiene are warranted.

\section{Acknowledgements}

The authors would like to thank all the subjects who participated this study, without whose kind co-operation, it would have been impossible to complete the research.

\section{THE AUTHORS}

\section{Dr. HV Amith}

BDS

Post Graduate Student,

Department of Preventive and Community Dentistry.

KLES Institute of Dental Sciences ,Belgaum, Karnataka.

\#29 C, II Main, 6th Cross, Bhaskar diary road,

Sadashivnagar, Belgaum 590010. Karnataka.

Mobile: +919448008007

E-mail: amith_hv@yahoo.co.in

\section{Dr. Anil V Ankola}

MDS

Professor \& Head, Department of Preventive and Community Dentistry. KLES Institute of Dental Sciences ,Belgaum, Karnataka.

\section{Dr. L Nagesh}

MDS

Ex-Professor and Head,

Department of Preventive and Community Dentistry.

KLES Institute of Dental Sciences ,Belgaum, Karnataka. 


\section{REFERENCES}

1. http://www.who.int/oral_health (accessed on 02-04-2006)

2. Tritten $\mathrm{CB}$, Armitage GC. Comparison of a sonic and a manual toothbrush for efficacy in supragingival plaque removal and reduction of gingivitis. J Clin Periodontol 1996;23:641-8.

3. Killoy WJ, Love JW, Love J, Fedi PF, Jr, Tira DE. The effectiveness of a counter-rotary Action Powered toothbrush and conventional toothbrush on Plaque Removal and gingival Bleeding. J Periodontol 1989; 60(8): 473-7.

4. Putt MS, Kleber CJ, Smith CE. Evaluation of an alum-containing mouthrinse in children for plaque and gingivitis inhibition during 4 weeks of supervised use. Pediatr Dent 1996;18:139-44.

5. Ram K S. Agnivesa's Charakasamhita Vol I(Sutra Sthana). Varanasi: Chowkhamba Sanskrit Series office; 2002:99-100, 123.

6. Ravi DT. Ashtanga Sangraha (Sutra Sthana) of Srimad Vrddhavagbhata. Delhi: Chawkhamba Sanskrit Pratishtan; 2003:551-4.

7. http://www.oilpulling.com/ (accessed on 21-12-2005)

8. Dingari LC. The Shalakya Tantra II.1st ed. Hyderabad: Smt D
Kamala Publishers; 2000:233-57.

9. Hiremath SS. Textbook of Preventive and Community Dentistry. New Delhi: Reed Elsevier India Private Ltd; 2007:

10. Peter S. Essentials of Preventive and Community Dentistry. 2nd ed. New Delhi: Arya (Medi) Publishing House; 2003:140-154.

11. Narayanaswamy K. Expel disease with an oily rinse. Vijay Times 2005 Nov 24; Health and Fitness. A:3.

12. Khalessi AM, Pack ARC, Thomson WM, Dunedin GRT. An in vivo study of the plaque control efficacy of Persica ${ }^{\mathrm{TM}}$ : a commercially available herbal mouthwash containing extracts of Salvadora persica. Int Dent J 2004;54:279-83.

13. Stoltze K, Bay L. Comparison of a manual and a new electric toothbrush for controlling plaque and gingivitis. J Clin Periodontol 1994;21:86-90.

14. Robertson PB, Armitage GA, Buchanan SA, Targgat EV. The design of trials to test the efficacy of plaque control agents for periodontal diseases in humans. J Dent Res 1989;68:1667-71.

15. Jeffcoat MK. Principles and Pitfalls of clinical trials-design. J Periodontol 1992;63:1045-51. 